\title{
A large area CsI RICH detector in ALICE at LHC
}

\author{
D. Di Bari ${ }^{\text {a* }}$ D. Cozza $^{\mathrm{a}}$, M. Davenport ${ }^{\mathrm{b}}$, A. Di Mauro ${ }^{\mathrm{b}}$, D. Elia ${ }^{\mathrm{a}}$, B. Ghidini ${ }^{\mathrm{a}}$, A. Grimaldi ${ }^{\mathrm{a}}$,

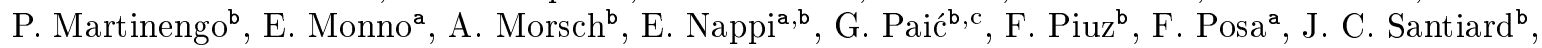 \\ S. Stucchi ${ }^{\mathrm{a}}$, G. Tomasicchio and T. D. Williams ${ }^{\mathrm{b}}$

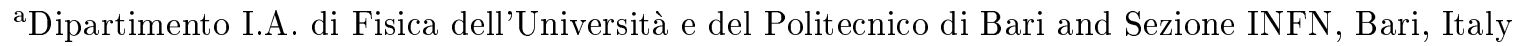 \\ ${ }^{\mathrm{b}}$ CERN, Geneva, Switzerland \\ ${ }^{\mathrm{c}}$ Ohio State University, Columbus, Ohio, USA
}

For the ALICE Collaboration

A $1 \mathrm{~m}^{2}$ CsI RICH prototype has been successfully tested in a hadron beam at CERN SPS. The prototype, fully equipped with $15 \mathrm{k}$ electronic channels, has been used to identify particles coming from $\pi$-Be interactions. Track reconstruction has been performed by using a telescope consisting of four gas pad chambers. A detailed description of the detector will be presented and results from test will be discussed.

\section{INTRODUCTION}

The ALICE (A Large Ion Collider Experiment) experiment[1] at LHC is dedicated to the study of the strongly interacting matter under extreme conditions of density and temperature where a new state of matter - the Quark-Gluon Plasma (QGP) - is predicted to be formed. Such conditions will be achieved at $\mathrm{LHC}$ in the $\mathrm{Pb}-\mathrm{Pb}$ collisions at a centre-of-mass energy of $2.75 \mathrm{TeV}$ per nucleon with a luminosity of about $10^{27} \mathrm{~cm}^{-2} \mathrm{~s}^{-1}$. These interactions are expected to be characterized by high multiplicities $(\sim 8000$ particles per unit of rapidity). The particle identification in ALICE plays an important role to determine inclusive particle ratios and transverse momentum spectra in the region dominated by the mini-jets of the pre-equilibrium stage in the ultrarelativistic nucleus-nucleus interactions. Therefore the identification of particles with high $\mathrm{p}_{t}$ will provide useful information on the jet quenching by measuring the ratio between protons and antiprotons yield. As shown in fig.1, the predicted pro-

\footnotetext{
${ }^{*}$ Corresponding author: Domenico.Dibari@ba.infn.it, fax
} no. $+39+805442470$ ton yield, for $\mathrm{p}_{t}>3 \mathrm{GeV} / \mathrm{c}$, will be about two times more than antiproton and therefore could be easily detected. In addition, the study of the particle correlations (HBT) at high $\mathrm{p}_{t}$ will give useful information about the size evolution of the emitting sourcei with momentum.

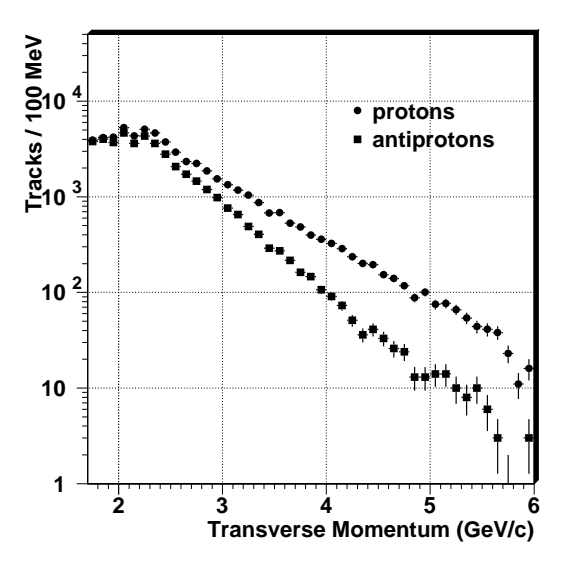

Figure 1. Expected proton and antiproton yields including jet quenching mechanism in central $\mathrm{Pb}-$ $\mathrm{Pb}$ collisions at LHC. 
A dedicated High Momentum Particle IDentification (HMPID) system, consisting of a single arm CsI-RICH based Cherenkov detector has been designed to identify charged particles $(\pi, K$ and $p$ ) in the $\mathrm{p}_{t}$ interval $2 \div 5 \mathrm{GeV} / \mathrm{c}$. In the ALICE experiment seven modules of $1.3 \times 1.3 \mathrm{~m}^{2}$ each will be placed on a barrel support $5 \mathrm{~m}$ far from the beam line, covering an acceptance of about $5 \%$ of the phase space, where a density of about 50 primary particles $/ \mathrm{m}^{2}$ is expected.

A large area CsI-RICH prototype (proto-2) of about $1 \mathrm{~m}^{2}$ has been built and tested at the CERN SPS H4 beam line with multiparticle events of high density obtained by interaction on target. The main results about the detector performance will be presented.

\section{THE CsI-RICH DETECTOR}

Technical details of the detector layout have been presented in previous papers [3-6]. Here a short description of the basic principle of functioning is reported. When a charged parti-

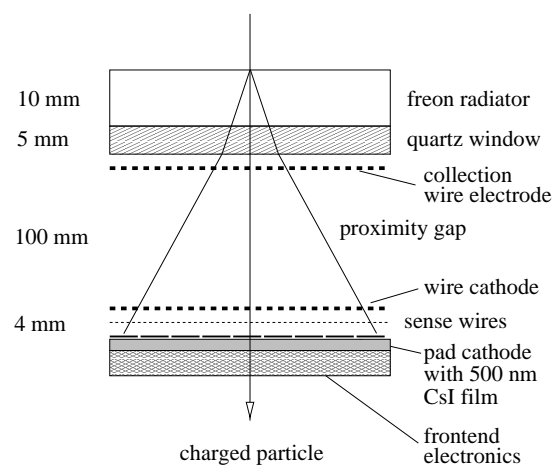

Figure 2. Schematic view of the HMPID CsIRICH.

cle moves through the RICH (fig.2), Cherenkov photons are emitted in the radiator consisting of $10 \mathrm{~mm}$ thick layer of $\mathrm{C}_{6} \mathrm{~F}_{14}$ liquid characterized by a low chromaticity. The refractive index determines a momentum threshold $\mathrm{p}_{t h} \simeq 1.26 \times m \mathrm{GeV} / \mathrm{c}$, where $m$ is the mass of the charged particle in $\mathrm{GeV} / \mathrm{c}^{2}$. The UV photons are detected by the photodetector, consisting of a conventional MWPC with anode wires of $20 \mu \mathrm{m}$ diameter, $4 \mathrm{~mm}$ pitch and $2 \mathrm{~mm}$ anode-cathode gap. They convert into electrons on a solid photocathode consisting of a thin layer $(500 \mathrm{~nm})$ of CsI evaporated onto a segmented pad plane of $8 \times 8$ $\mathrm{mm}^{2}$.

The detector volume ("proximity gap") between the radiator and the MWPC ensures a more convenient size of Cherenkov ring images on the photocathode plane. The collection wire electrodes placed in the proximity gap, near the quartz window, avoid the electrons released by ionizing particles to enter in the MWPC sensitive volume. The analog pad readout allows to obtain an accurate localization by means of the reconstruction of centroids.

A prototype, corresponding to $2 / 3$ of the final RICH module, has been built using four large photocathodes (PC29-32) of $64 \times 40 \mathrm{~cm}^{2}$. A first test of the detector fully equipped with the four PCs and one full-size-radiator tray was performed at the SPS in November 1997. Subsequently, two SPS tests were performed in April and July 1998, the latter one with the complete radiator composed of two trays.

\section{CERN SPS TEST BEAM RESULTS}

Fig.3 shows the experimental setup. A scintillator doublet was placed in the beam upstream of the target to provide the beam coincidence in a sensitive area of $5 \times 5 \mathrm{~mm}^{2}$. A veto array, con-

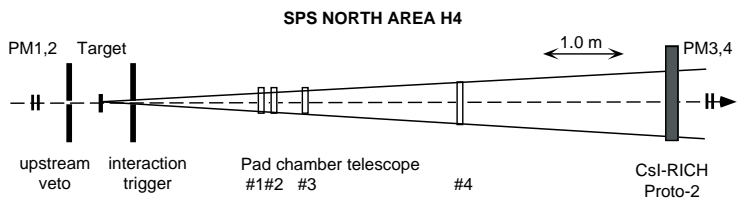

Figure 3. Experimental layout used at the SPS/H4 test beam. 
sisting of four scintillators $20 \times 20 \mathrm{~cm}^{2}$ providing two- or four-fold coincidences as veto signals, was used to veto upstream interactions. A pion beam (along the $\mathrm{Z}$ axis) of $350 \mathrm{GeV} / \mathrm{c}$ was incident on a berillium target. An interaction array, identical to the veto array, provided various interaction trigger conditions.

Table 1

Geometrical parameters of the telescope pad chambers

\begin{tabular}{ccccccc}
\hline MWPC & $\begin{array}{c}\text { Anode } \\
\text { wire }\end{array}$ & $\begin{array}{c}\text { Coord. } \\
\text { centroid }\end{array}$ & $\begin{array}{c}\text { Pad size } \\
\left(\mathrm{mm}^{2}\right)\end{array}$ & \multicolumn{3}{c}{$\begin{array}{c}\text { Pad cathode } \\
\text { size }(\text { pads })\end{array}$} \\
\hline 1 & vertical & $\mathrm{Y}$ & $8 \times 8$ & 30 & $\times$ & $\mathrm{Y}$ \\
2 & horiz. & $\mathrm{X}$ & $8 \times 8$ & 32 & $\times$ & 30 \\
3 & horiz. & $\mathrm{X}$ & $8 \times 8$ & 40 & $\times$ & 36 \\
4 & horiz. & $\mathrm{X}$ & $8 \times 8$ & 56 & $\times$ & 60 \\
\hline
\end{tabular}

Four pad chambers were used as tracking devices to detect the impacts of the particles produced in the interaction. The acceptance of the tracking telescope covers only a half of the sensitive area of the RICH detector. In table 1 the geometrical parameters of the tracking chambers are summarized.

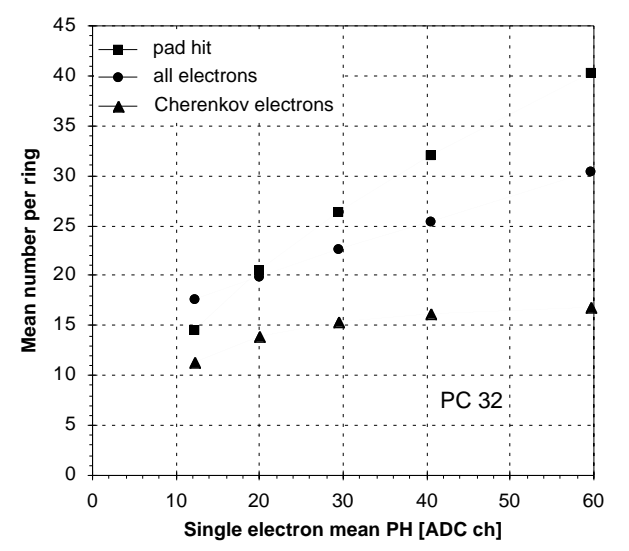

Figure 4. Distributions of the mean number, per ring, of pad hits $\left(N_{\text {pad }}\right)$, electrons $\left(N_{\text {tot }}\right)$ and Cherenkov photoelectrons $\left(N_{\text {res }}\right)$ as a function of the single-electron mean pulse height.
The large proto-2 RICH detector was located on a movable stage with remote control in horizontal and vertical motions. The anode wires were aligned along the horizontal ( $\mathrm{X}$ axis). Finally, a second scintillator doublet providing beam coincidence with a sensitive area of $5 \times$ $5 \mathrm{~mm}^{2}$, was placed downstream from the RICH.

Fig.4 shows the distributions of the mean number $N_{\text {pad }}$ of pad hits, electrons $N_{\text {tot }}$ and $N_{\text {res }}$ per ring as a function of single photoelectron mean pulse height (in unit of ADC channels; 1 ADC ch. $=0.17 \mathrm{fC}$ ). While $N_{t o t}$ increases almost linearly with the chamber gain, due to the contribution of the feedback photons which increases linearly with the chamber gain, $N_{\text {res }}$ saturates to a value of 15-16 Cherenkov photoelectrons per ring.

This dependence can be explained by the fact that the feedback photons emitted from the primary avalanches have a high probability to impact on the same pad as the primary one, because of the small chamber gap ( $2 \mathrm{~mm})$ compared to the pad size $\left(8 \times 8 \mathrm{~mm}^{2}\right)$. This effect improves the

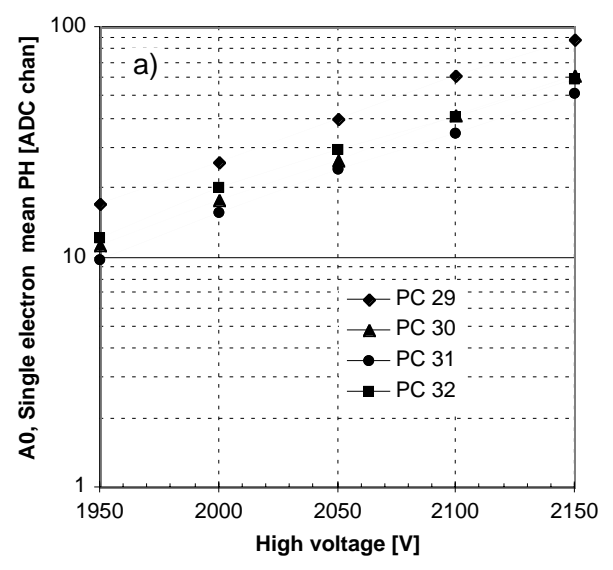

Figure 5. Mean single-electron pulse height as a function of high voltage measured at the centre of each of the four photocathodes.

detection efficiency, although increases also the pad pattern occupancy by enlarging the size of the clusters and their number. This is shown in 
fig.4, where $N_{\text {pad }}$ increases as a function of the chamber gain.

Fig.5 shows the photodetector gain $\mathrm{A}_{0}$ as a function of the chamber voltage when the beam was centred at the middle of each photocathode (the curves for PC29 and PC31 have been shifted by $-30 \mathrm{~V}$ and $+30 \mathrm{~V}$, respectively, due to a non uniformity of $\sim 120 \mu \mathrm{m}$ with respect to the nominal anode-chatode gap).

Tests to evaluate the gain and photon yield uniformity have been performed. Information about the gain uniformity has been obtained by taking single track runs moving the ring centre across the photocathode plane. Fig.6 shows, for one photocathode (PC32), the photon pad regions divided in four quadrants, for three different beam positions. The numbers in each quadrant, corresponding to the mean pulse height, indicate a good uniformity of the chamber gain.

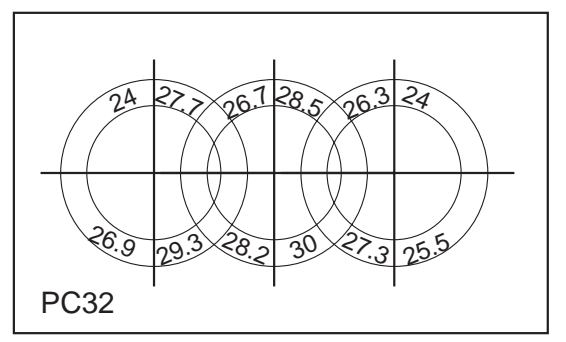

Figure 6. Evaluation of the uniformity of the chamber gain for the photocathode PC32. The numbers correspond to the mean pulse height spectrum measured in $90^{\circ}$ sectors of the Cherenkov fiducial zones $(\mathrm{HV}=2050 \mathrm{~V})$.

Fig.7 shows an example of the azimuthal distribution of the photon pads in the Cherenkov fiducial zone, indicating a uniform response all around the ring. The fluctuations around $0^{\circ}$, $180^{\circ}$ and $360^{\circ}$ are due to the size of the pad giving discrete value of the azimuth greater than the bin size $\left(1^{\circ}\right)$. A good uniformity (within $10 \%$ ) of the response of the four photocathodes have been checked over their whole area.

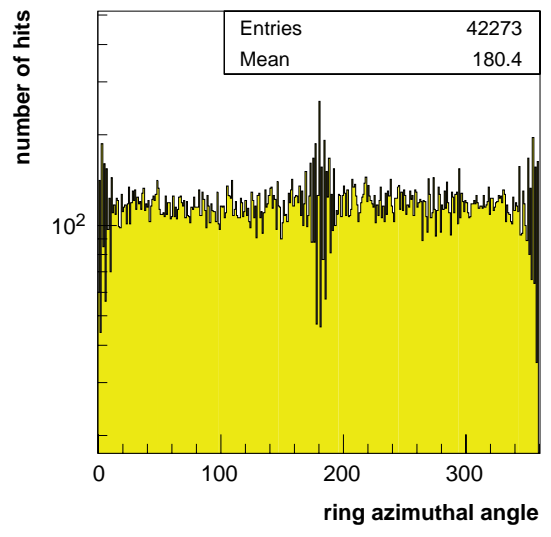

Figure 7. Azimuthal distribution of the photon pad hits in the Cherenkov fiducial zone $(\mathrm{HV}=2050 \mathrm{~V})$.

Single beam events at the SPS have been processed in order to obtain the Cherenkov angle resolution in the absence of background coming from other tracks. Fig. 8 shows the distribu-
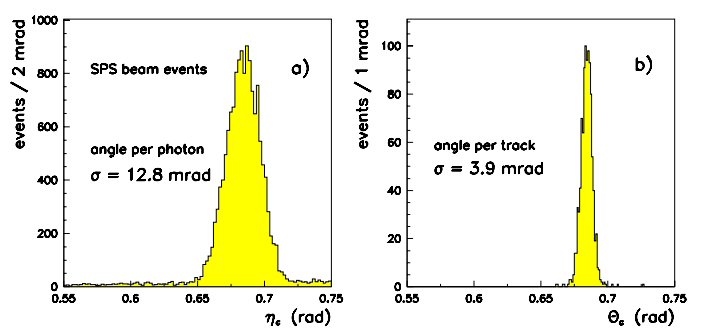

Figure 8. Photon angle (a) and track Cherenkov angle (b) distributions for beam events at the SPS. The distribution (b) has been obtained considering only photons with $0.55<\eta_{c}<0.75 \mathrm{rad}$ to shorten the computing time.

tion of extracted Cherenkov angles per cluster where a clear signal with negligible background can be seen (details about the method to obtain 
the Cherenkov angle from the pad information can be found in [4]). A Gaussian fit of the distribution gives a $\sigma_{\eta_{c}} \sim 12.8 \mathrm{mrad}$. By averaging the $\eta_{c}$ for all the photons, the mean Cherenkov angle per track $\theta_{c}$ is obtained: the distribution, shown in fig. $8 \mathrm{~b}$, indicates this resolution to be $3.9 \mathrm{mrad}$.

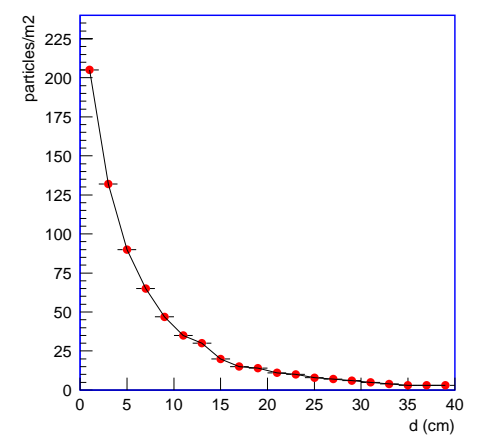

Figure 9. Track density on the HMPID cathode plane in real $350 \mathrm{GeV} / \mathrm{c} \pi^{-}$-Be events.

During the test, $\pi$-Be interactions have also been collected to study the performance of the RICH detector in multiparticle events. Since no sweeping magnet has been employed in the test, the emitted particles are defining a very narrow cone. The distribution of the track density, in the RICH, as a function of the distance from the beam line, is shown in fig. 9. Although densities greater than 50 particles $/ \mathrm{m}^{2}$ could be reached for $\mathrm{d}<10 \mathrm{~cm}$, the performance of the RICH has been found stable and the detector operation satisfactory. Fig. 10 shows a three dimensional display of one $\pi$-Be event. Since the acceptance of the tracking telescope covers only a fraction of the whole RICH surface, only a fraction of the rings could be associated to the reconstructed tracks.

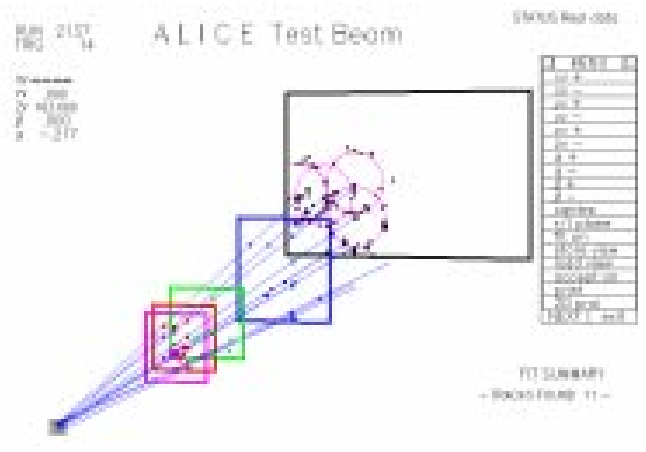

Figure 10. Three dimensional display of an SPS $350 \mathrm{GeV} / \mathrm{c} \pi^{-}$-Be event. Eleven tracks are reconstructed in the telescope by requiring one hit on each pad chamber to reconstruct a track. Rings corresponding to $\beta=1$ particles are drawn to guide the eyes.

\section{CONCLUSION}

In the ALICE experiment, a $12 \mathrm{~m}^{2}$ array consisting of seven proximity focused $\mathrm{C}_{6} \mathrm{~F}_{14}$ liquid radiator RICH modules will be implemented for particle identification at high momentum. A large area CsI-RICH prototype, corresponding to $2 / 3$ of one final RICH module, has been successfully tested at CERN SPS test beam, showing a good performance during operation even in events with high particle density. No maintenance was needed during the run period.

\section{REFERENCES}

1. Alice Collaboration, Technical Proposal, CERN/LHCC 95-71.

2. Alice Collaboration, Addendum to the Technical Proposal, CERN/LHCC 96-32.

3. F. Piuz, Nucl. Instr. and Meth. A371(1996) 96.

4. ALICE Collaboration, Technical Design and Report, Detector for High Momentum PID, CERN/LHCC 98-19.

5. E. Nappi et al., High Momentum PID with $\mathrm{RICH}$ detectors in the ALICE experiment at LHC,28th Int. Conference on High Energy Physics. ICHEP96-Warsaw 1996, World Scientific, Singapore 1997 (1714-1718).

6. E. Nappi et al., Internal Note ALICE 98-20. 